\title{
$\chi-\gamma$ mixing in an expanding Universe
}

\section{Subhayan Mandal*i}

Affiliation - University of Liège

E-mail: smanda1dulg.ac.be

\section{Jean-René Cudell}

Affiliation - University of Liège

E-mail: br.cudel1dulg.ac.be

\begin{abstract}
We establish the equation of motion of pseudoscalar particles coupled to an electromagnetic field in a classical gravitational background through the use of conformal time and flat geometry. We show that the expansion of the universe can lead to larger mixing than in a stationary universe. We also show that for a broad range of parameters, one can obtain resonant mixing, i.e. a region in which the mixing becomes maximum.
\end{abstract}

The 2011 Europhysics Conference on High Energy Physics-HEP 2011,

July 21-27, 2011

Grenoble, Rhône-Alpes France

\footnotetext{
* Speaker.

${ }^{\dagger}$ Poster-Session
} 


\section{Introduction}

Hypothetical pseudoscalar particles [ [] mix with photons [] while propagating through a background magnetic field. This can lead to observable effects in propagating photon properties [B] such as birefringence [四] and dichroism [四]. Recent observations of polarised light in the optical band, coming from distant quasars, is also very suggestive of these phenomena [5]. Here we shall delineate a scenario where these effects can be maximised through resonance.

\subsection{Methodology}

In this note we shall briefly describe our work of resonant mixing with classical gravity acting as a background. Firstly it is assumed that the universe is expanding and is flat. Next, the metric is $\operatorname{diag}\left(1,-a^{2}(t),-a^{2}(t),-a^{2}(t)\right)$ and not the Minkowski one. For the ease of computation we have switched to the conformal time parameter. This facilitates the following :-

1. The scaled metric resembles the Minkowski one. The scale factor depends only on time

2. One can use the normal prescription of writing the action with background gravity

3. A few changes of variables make the equations look less formidable.

4. Approximate solution to the coupled system are obtained expressly.

\section{Result}

The mixing angle for the mixing of pseudoscalars with photons has been found to be :-

$$
\theta=\frac{1}{2} \tan ^{-1}\left[\frac{2 k B p_{\chi}}{a(\eta)\left(m^{2} a^{2}(\eta)-\omega_{p}^{2}(\eta)\right.}\right]
$$

This shows that there is a non trivial $(a \neq 0)$ resonance point for mixing. When the scale factor is equal to the ratio of plasma frequency to pseudoscalar mass, the mixing phenomena peaks to its maximum value; as shown in fig: $\mathbf{m}$.

\section{Conclusion}

Although the primary objective is fulfilled by the result it should be noted that this result is not found in the radio wavelength, and there has been no observation in X-ray or higher frequency bands. However our preliminary study shows that photons from the furthest (more distant than the quasars) corners of the universe should be polarised upon reaching the earth or the observer. Failing which should impy any, or both , of the following.

1. Either the non-existence very low mass ALP's

2. Or the non existence of nearby intersteller magnetic field.

However, recent studies On GRB spectra show departure from synchroton radiation and signature of photon-ALP mixing [目], and similar remarks are made by studying their empirically established luminosity relations [प]. 


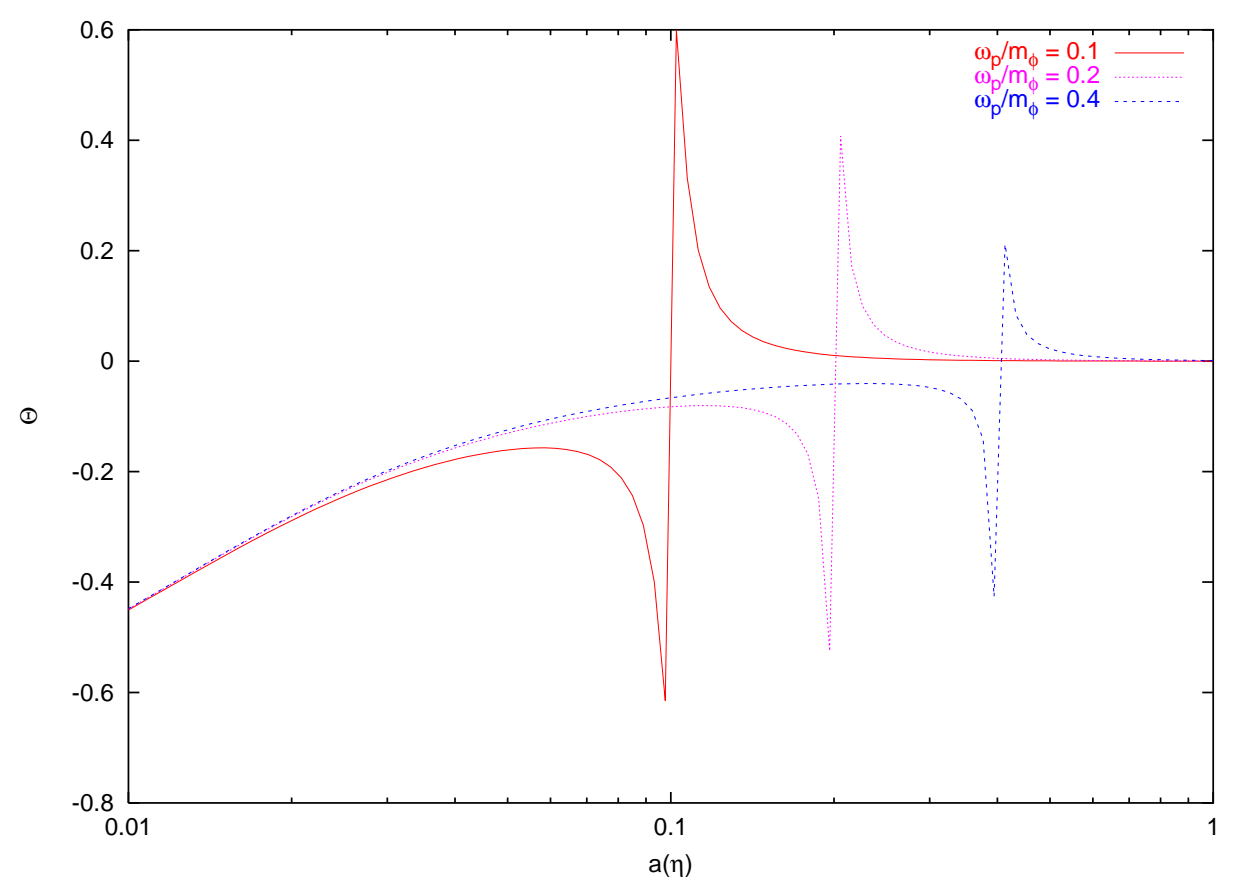

Figure 1: Mixing Angle WRT scale factor.

\section{References}

[1] S. Weinberg, Phys. Rev. Lett. 40, 223, (1978);

F. Wilczek, Phys. Rev. Lett. 40, 279, (1978).

[2] P. Sikivie, Phys. Rev. D 32, 2988, (1985)

[3] S. Das et. al. J. Cosmol. Astropart. Phys., 0506, (2005), 002

[4] J. A. Beswick \& C. Rizzo arXiv:quant-ph/0702128

[5] D. Hutsemékers, R. Cabanac, H. Lamy and D. Sluse, Astronomy and Astrophysics 441, 915, (2005)

[6] O. Mena, S. Razzaque \& F. Villaecusa-Navarro, arXiv:1101.1903

[7] C. Burrage, Nuc. Phys B 194, 190-195, (2009) 